\title{
HOMEOMORPHISMS WITH MANY RECURRENT POINTS
}

\author{
BENJAMIN HALPERN
}

\begin{abstract}
Let $X$ be a topological space and $H(X)$ the space of all homeomorphisms of $X$ onto itself with the compact open topology. If $f \in H(X)$ and $p \in X$, then $p$ is a recurrent point of $f$ provided $p$ is in the closure of $\left\{f^{n}(p) \mid n \geqslant 1\right\}$. It is shown that if $X$ is Hausdorff and $V$ is a nonempty open subset of $X$ homeomorphic to Euclidean $n$-dimensional space with $n \geqslant 1$, then $\{f \in H(X) \mid$ the recurrent points of $f$ are dense in $V\}$ is nowhere dense in $H(X)$.
\end{abstract}

Let $X$ be a topological space and $H(X)$ the space of all homeomorphisms of $X$ onto itself with the compact open topology. If $f \in H(X)$ and $p \in X$, then $p$ is a recurrent point of $f$ provided $p \in\left\{\overline{f^{n}(p) \mid n \geqslant 1}\right.$, where $\bar{A}$ denotes the closure of $A$. Given a fixed Borel measure $\mu$ on $X$ which assigns a positive value to each nonempty open subset of $X$, we say an $f \in H(X)$ is recurrent (with respect to $\mu$ ) provided the set of nonrecurrent points of $f$ has $\mu$-measure zero. The following theorem is proven in [1].

THEOREM 1. If $X$ is a compact manifold without boundary, of dimension greater than zero and of nonzero Euler characteristic, then the set of all recurrent homeomorphisms of $X$ is nowhere dense in $H(X)$.

We establish here a stronger conclusion than that of Theorem 1 while removing the hypotheses that $X$ is compact and has nonzero Euler characteristic. We also weaken the hypothesis that $X$ is an $n$-dimensional manifold without boundary, $n \geqslant 1$, to the assumption that there is a nonempty open subset of $X$ homeomorphic to some Euclidean space $\mathbf{R}_{n}$ with $n \geqslant 1$. The proof is a modification of the argument in [1].

THEOREM 2. If $X$ is a Hausdorff space and $V$ is an open subset of $X$ homeomorphic to $\mathbf{R}^{n}$ with $n \geqslant 1$, then $R=\{f \in H(X) \mid$ the recurrent points of $f$ are dense in $V\}$ is nowhere dense in $H(X)$.

Proof. Assume the hypothesis and the contrary to the conclusion. Then there is a nonempty open set $W \subset H(X)$ such that $W \subset \bar{R}$. Let $f \in W \cap R$. Then the recurrent points of $f$ are dense in $V$. Let $p$ be one.

Received by the editors September 8, 1975.

AMS (MOS) subject classifications (1970). Primary 58D99; Secondary 22A65.

Key words and phrases. Homeomorphism, recurrent point, manifold, compact open topology. 
Claim. There exists a $\varphi \in W$ such that $\varphi^{k}(p)=p$ for some $k \geqslant 1$.

Proof. Since $V$ is homeomorphic to $\mathbf{R}^{n}, n \geqslant 1$, we identify $V$ with $\mathbf{R}^{n}$. Let $\varepsilon$ be any positive number. Since $p$ is a recurrent point for $f, f^{k}(p) \in U_{\varepsilon}$ $=\left\{q \in V=\mathbf{R}^{n} \mid\|q-p\|<\varepsilon\right\}$ (where $\|\cdot\|$ is the Euclidean norm) for some $k \geqslant 1$. Let $k$ be the smallest such integer. Let $\alpha$ be a homeomorphism of the closed ball $\bar{U}_{\varepsilon}=\{q \in V \mid\|q-p\| \leqslant \varepsilon\}$ onto itself such that $\alpha$ is fixed on the sphere $S_{\varepsilon}=\{q \in V \mid\|q-p\|=\varepsilon\}$ and $\alpha\left(f^{k}(p)\right)=p$. Extend $\alpha$ to a homeomorphism of $X$ onto itself by setting $\alpha(x)=x$ for $x \notin U_{\varepsilon}$. Set $\varphi=\alpha \circ f$. It is immediate that $\varphi^{i}(p)=f^{i}(p)$ for $0 \leqslant i<k$ and $\varphi^{k}(p)=p$. It is not hard to see that for $\varepsilon$ sufficiently small, $\varphi \in W$. Indeed, for the special case of $W=N(C, O)=\{h \in H(X) \mid h(C) \subset O\}$, where $C$ is compact and $O$ is open, we have $\varphi \in W$ whenever

$$
\varepsilon<\min \left(1, \frac{1}{2} \text { the distance from } f(C) \cap \bar{U}_{1} \text { to } \mathbf{R}^{n}-O \cap U_{2}\right) .
$$

The general case follows because the sets $N(C, O)$ generate the compact open topology. This proves the claim.

The rest of the proof follows the proof in [1].

Let $k$ be the smallest positive integer such that $\varphi^{k}(p)=p$. Pick an $\varepsilon>0$ such that $U_{\varepsilon}, \varphi\left(U_{\varepsilon}\right), \varphi^{2}\left(U_{\varepsilon}\right), \ldots, \varphi^{k-1}\left(U_{\varepsilon}\right)$ are pairwise disjoint. Pick a $\tau, 0$ $<\tau<\varepsilon / 3$ such that $\varphi^{k}\left(U_{\tau}\right) \subset U_{\varepsilon / 3}$. Let $\eta$ be a homeomorphism from $\bar{U}_{\varepsilon}$ onto itself such that $\eta$ is fixed on $S_{\varepsilon}$ and $\eta\left(\bar{U}_{2 \varepsilon / 3}\right) \subset U_{\tau / 2}$. See [1] for a detailed construction. Extend $\eta$ to a homeomorphism of $X$ onto itself by setting $\eta(x)=x$ for $x \notin U_{\varepsilon}$. With an argument similar to that above it is not hard to see that for $\varepsilon$ sufficiently small, $g=\varphi \circ \eta \in W$.

For $k \geqslant 2$ pick $t_{i}, i=1,2, \ldots, k$, such that $\frac{1}{2} \tau=t_{1}<t_{2}<\cdots<t_{k}$ $=\tau$. For all $k \geqslant 1$ set $N_{1}=N\left(\bar{U}_{2 \varepsilon / 3}, \varphi\left(U_{\tau / 2}\right)\right)$ and

$$
N_{i}=N\left(\varphi^{i-1}\left(\bar{U}_{t_{i-1}}\right), \varphi^{i}\left(U_{t_{i}}\right)\right)
$$

for all $i, 2 \leqslant i \leqslant k$. Next, set $N=\cap_{i=1}^{k} N_{i}$. Unravelling the definition of the basic open set $N$, we see that if $h \in N$ and $q \in U_{2 \varepsilon / 3}-\bar{U}_{\varepsilon / 3}$ (which is nonempty because $n \geqslant 1$ ), then $q$ is not a recurrent point of $h$. This also uses the fact that $U_{\varepsilon}, \varphi\left(U_{\varepsilon}\right), \ldots, \varphi^{k-1}\left(U_{\varepsilon}\right)$ are pairwise disjoint. This shows that $N \cap R=\varnothing$. But $g \in N$ and so $g \notin \bar{R}$. Yet, $g \in W \subset \bar{R}$, a contradiction. Q.E.D.

\section{REFERENCES}

1. Chung-wu Ho, On the homeomorphisms which satisfy the Poincaré Recurrence Iheorem, Proc. Amer. Math. Soc. 58 (1976), 272-276.

Department of Mathematics, Indiana University, Bloomington, Indiana 47401 